\title{
Melanin as an Endogenous MRI Contrast Agent for Stem Cell Delivery in Stroke
}

Randy Chang ( $\nabla$ rochang@mednet.ucla.edu )

University of California, Los Angeles

Jesse Jones

University of California, Los Angeles

\section{Research Article}

Keywords: Melanin, Endogenous MRI, Stem Cell Delivery, Stroke, Contrast Agent

Posted Date: April 30th, 2021

DOl: https://doi.org/10.21203/rs.3.rs-466208/v1

License: (c) (1) This work is licensed under a Creative Commons Attribution 4.0 International License. Read Full License 


\section{Abstract}

In vivo measures of survival, growth, and migration of stem cell transplants for stroke therapy remain inadequate. Since such biomarkers in the central nervous system do not exist, we sought to investigate the potential of melanin as an endogenous MR contrast medium for monitoring cell lineage transplants. Expression of a human gene, tyrosinase, induces melanogenesis, but unlike particle-based tracking agents, tyrosinase incorporated into the host genome will be replicated during mitosis.

Tyrosinase and the gene for a co-enzyme, tyrosinase-related protein 1, were placed under the CMV promoter. The construct was inserted into 293 HEK (human embryonal kidney) and iPS NPC (induced pluripotent neural progenitor) cells via plasmid transfection and viral transduction, respectively. Stroked mice were injected with melanin-producing cells. Control mice were injected with native cell lines. In vitro expression was measured by fluorescent microscopy, immunocytochemistry, spectroscopy, PCR, and MRI.

Robust in vitro melanin production was achieved in both cell lineages demonstrated by significant T1 shortening on in vivo MRI. Pathologic correlation demonstrated colocalization of pigmented regions in the injection sites with human antinuclear antibody staining. Through induction of melanogenesis that is reproducible across multiple cell divisions, MR-based imaging of clinically relevant cell lineage transplants is possible.

\section{Introduction}

Stem cell delivery is a promising therapy in stroke as well as in neurodegenerative disorders [1-3]. However, in vivo measures of survival, growth, and migration of cell transplants remain inadequate. Unlike a stem cell therapy in other organ systems, there are substantial limitations to tracking stem cell treatments in the brain and spinal cord after transplant-prohibiting an in vivo biomarker of these therapies [4]. Image-based tracking approaches have focused on MRI given its superb soft tissue contrast and commonplace clinical use. Ferrous intracellular compounds such as iron nanoparticles, among the first such agents, produce negative contrast that may be confused with susceptibility artifact from hemorrhage or surgery [5, 6]. These techniques quantify loss of signal in the T2 sequence [7]. Positive contrast agents like gadolinium nanoparticles obviate these particular shortcomings, but as with any particles, suffer from signal dilution with every mitosis [8]. We therefore sought to develop a more durable positive contrast agent whose signal intensity correlates with cell number [9].

Melanin is an endogenous pigment synthesized in neurons throughout the brain [10] that scavenges heavy metals and free radicals. Its accumulation within the normal human substantia nigra is seen on 3.0 Tesla MRI as foci of T1 shortening [11-13] due to paramagnetic effects imparted by bound iron and other metallic cations. Visualization of melanin in physiologic concentrations suggests it may serve as a low toxicity contrast agent. Insertion of a single gene, tyrosinase (TYR), induces melanogenesis in several human cell lines [14-17]. Unlike particle-based tracking agents, TYR incorporated into the host genome will be replicated during each cell division. 
We demonstrate the feasibility of expressing tyrosinase in induced pluripotent stem neural precursor (iPSNPC) cells as MRI tracking agents in stroked mouse brain. These findings may support further research into cell tracking for human clinical trials.

\section{Methods}

This pilot study aimed to demonstrate a quantifiable MRI signal from melanin production, through gene delivery in a cell lineage of potential use in stroke therapy. Therefore, the gene construct was designed with a strong, constitutive promotor to drive expression over the course of the experiment. Initial studies were performed in 293 HEK cells given their accessibility and ease of use. Then iPS NPCs, a lineage with potential clinical relevance, were assessed through similar experiments. HEK cells were sourced from Carmichael Lab at University of California, Los Angeles, and iPS NPC cells were sourced from Lowry Lab at University of California, Los Angeles.

\section{Gene construct}

Human tyrosinase (TYR, NM_000372.3) and tyrosinase-related protein 1 (TYRP1, NM_000550) sequences were acquired from OriGene (Rockville, MD) (cat.no. SC300059 and SC119834, respectively). PCDH-CMVTYRP1-2A-copGFP was digested with Notl and Sall and EF1-TYR-2A-copGFP was released from pCDHEF1-TYR-2A-copGFP by Notl and Sall digestion. Released EF1-TYR-2A-copGFP fragment was then cloned into PCDH-CMV-TYRP1. Constructs were restriction digested and sequenced to confirm identity and tested in vitro for melanin production.

\section{Cell Culture}

293 HEK cells were cultured in medium composed of DMEM, 10\% fetal bovine serum, glutumax, and pyruvate (Gibco; Waltham, MA). iPS NPC media consisted of DMEM/F12 (Gibco), B27 and N2 supplements (Gibco; Waltham, MA), fibroblast growth factor and epidermal growth factor (Invitrogen; Waltham, MA). 293 HEK cells were plated at a density of $3.5 \times 106$ and transfected with TYR-TRP-GFP

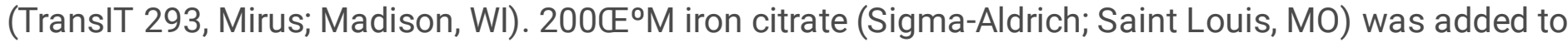
the culture medium 1 day following transfection to simulate in vivo conditions and provide a substrate for melanin scavenging. iPS NPCs were plated at a density of $5 \times 104 \mathrm{~cm} 2$ and transduced with TYRTYRP-GFP using lentivirus. Lentivirus was constructed and packaged using standard approaches $[18,19]$.

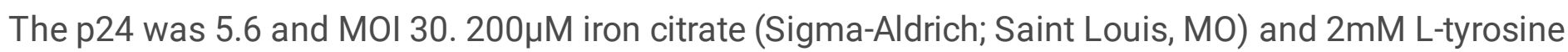
(Sigma-Aldrich; Saint Louis, MO) were added to the culture medium 3 days following transduction to account for slower initiation of expression with virus than particles.

Assessment of Melanogenesis

293-HEK and iPS-NPC transgene expression was evaluated qualitatively during cell culture by epifluorescence light microscopy of culture flasks. At 5 days post-transfection, 106 293-HEK cells were resuspended in $1 \mathrm{ml}$ PBS for SPECT. Absorbance was measured from 300 to $600 \mathrm{~nm}$. At 7 days post- 
transfection, iPS-NPCs were harvested for RT-PCR (Invitrogen; Waltham, MA). Forward and reverse TYR primers (Valuegene; San Diego, CA) were: GCGGGATGCAGAAAAGTGTG and TCGGCTACAGACAATCTGCC. RT-PCR products were run on a 1.2\% agarose gel (Sigma-Aldrich; Saint Louis, MO). Additionally, iPS-NPC's transduced and cultured in a 24 well plate were assessed on posttransduction day 7 with immunocytochemical (mouse anti-human tyrosinase antibody, Abcam; Cambridge, MA) and Fontana-Mason (Abcam; Cambridge, MA) stains. Control groups were composed of nontransfected iPS-NP cells cultured in iron and L-tyrosine.

In vitro MRI

293-HEK cells were harvested on post-transfection day 5 and mixed with nontransfected cells in ratios of $0: 1,1: 4,1: 1$, and $1: 0$ for a total population of $5 \times 106$ cells. Utilizing a constant cell number eliminates cell settling as a variable in image analysis. Cells were centrifuged and resuspended in $100 \mu$ low melting agarose (Sigma-Aldrich; Saint Louis, MO). Cell containing agar was sandwiched between layers of plain

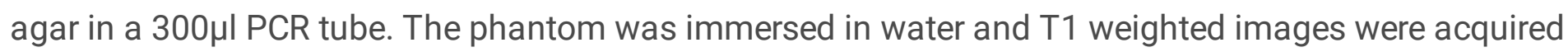
on a 3T MRI scanner (Siemens; Ehlangen, Germany).

Photothrombotic stroke (PTS) and intracerebral cell (IC) injection

All experimental protocols were approved by the Animal Research Committee (ARC) at University of California, Los Angeles, and performed in accordance with relevant named guidelines and regulations of the ARC. The authors have complied with ARRIVE guidelines.

11 NOD scid gamma (NSG) mice initially underwent photothrombotic stroke [20]. Subsequently:

1 mouse underwent MRI 7 days after stroke to pilot injection targeting. The brain was stained with tetrazolium chloride (TTC) at this time point to confirm cerebral infarction and also to measure infarct size in preparation for intracerebral cell injections.

On post-stroke day 5, intracerebral 293-HEK cell injections were administered adjacent to the infarct in 10 mice:

5 mice received 293-HEK cells transfected with TYR-TYRP-GFP cultured in iron for 3 days. 5 control group mice received non-transfected 293-HEK cells cultured with iron for 3 days.

225,000 293-HEK cells in $3 \mu \mathrm{l} \mathrm{PBS}$ were injected $1 \mathrm{~mm}$ anterior, posterior, and lateral to the stroke isocenter (775,000 cells/mouse).

Steps (b) through (d) were repeated with iPS-NPCs in 10 mice.

In vivo MRI

1 day after intracerebral injection mice were anesthetized and imaged on a 7T MRI (Bruker; Billerica, MA) utilizing coronal T1, T2 and inversion recovery T1 mapping sequences. 
Immunohistochemistry

18 mice were sacrificed 1 day following MRI. Mice were perfused with $4 \%$ paraformaldehyde, and sectioned in coronal plane. Slide-mounted frozen sections were stained with human nuclear ( $\mathrm{HuNu}$, Abcam; Cambridge, MA), glial fibrillary acidic protein (GFAP, Abcam; Cambridge, MA) and feminizing locus on X-3 (NeuN, Abcam; Cambridge, MA) antibodies as well as 4',6-diamidino-2-phenylindole (DAPI, Thermo Fisher Scientific; Waltham, MA). Slides were imaged on a confocal microscope (Nikon; Melville, NY).

\section{Statistical Analysis}

Descriptive measures and summary statistics were performed using SPSS software version 20.0 [21]. In addition, the Mann-Whitney test and Students' t-test were performed and significance was considered as two-sided $\mathrm{p}$-value $<=0.05$.

\section{Results}

Melanogenesis

Epifluorescence performed one day after transfection demonstrated higher GFP signal than background in approximately $50 \%$ of 293-HEK cells (Figure I). TYR TRP 293 HEK cell absorbance at $350 \mathrm{~nm}$ was 0.45 compared to 0.14 for control cells $(p=0.001)$, demonstrating significantly increased melanin pigmentation in the transfected group. T1 shortening was present in all $293 \mathrm{HEK}$ cell dilutions containing transfected cells, whereas no significant increased T1 signal was present in vials with non-transfected cells alone $(p<0.001)$ (Figure II).

iPS-NPC's did not exhibit fluorescence following viral transduction. Therefore, additional tests for construct expression were performed. Gel electrophoresis of transduced iPS-NPC RT-PCR products detected tyrosinase mRNA in transfected cells. This was absent in non-transfected cells, indicating successful transfection (Figure III). iPS-NPC's transduced and cultured in a 24 well plate and stained with anti-tyrosinase antibody on post-transduction day 7 demonstrated cytosolic tyrosinase protein in approximately $30 \%$ of cells. Positive Fontana-Mason staining for melanin (Figure IV), confirmed that translation of tyrosinase resulted in tangible melanogenesis.

In vivo MRI

T1 shortening was present in all mice 1 day after TYR-293 HEK IC injection. Mean T1 value in a region of interest around the injection sites was $960.2 \mathrm{msec}$, compared to $1392.8 \mathrm{msec}$ for nontransfected 293 HEK cells $(p=0.009)$. T1 shortening was also present in all mice 1 day after TYR TRP iPS-NP IC injection. Mean T1 value in a region of interest around the injection sites was $1041 \mathrm{msec}$ compared to $1363 \mathrm{msec}$ for nontransfected iPS-NPC's $(p=0.014)$ (Figure V).

Immunohistochemistry 
Specimens through photothrombotic stroke demonstrated a sharply demarcated border between the infarct and astrocyte and neuronal cells. Slices through transfected 293-HEK and transduced iPS-NPC IC injections collected 1 day after initial MRI were HuNu positive (Figure VI). These regions co-localize with dark melanic pigment under light microscopy.

\section{Discussion}

All newly developed human therapies need some element of post-delivery monitoring to assess appropriate bioavailability and establish causal dose-response relationship of treatment effects. For pharmacological agents, this is relatively straightforward in the monitoring of drug levels. However, once cell lines or products are delivered into the human brain, their migration and survival are unknown. Without adequate tracking methods, the fate of transplanted cells, the degree of migration and the relationship of dose to survival and recovery cannot be determined. As these are core issues of any developing human therapy [22], the inability to track transplanted cells will impact the effectiveness of human trials.

The ideal tracking agent for monitoring cell therapy is nontoxic, simple to measure, and highly correlated with transplant population size, location, and health [23]. Here, we show preliminary evidence that transgenic expression of TYR and TYRP1 could satisfy many of these conditions. Neuromelanin is a scavenger produced endogenously in neurons throughout the human brain, where it is stored in autophagic vacuoles [10]. Iron bound melanin is paramagnetic, creating a strong T1 shortening effect that lends itself to visualization by MRI at physiologic concentrations [11-13].

TYR and TYRP1 transgene delivery represents the simplest method of inducible melanogenesis and allows for regulated expression at the promotor level to reduce toxicity in vivo [15-17]. We have demonstrated the feasibility of labeling human cells, including biologically relevant iPS-NPC's [28, 29]. Moreover, these cells were transplanted in a similar population size and peri-infarct location as employed in recent human stroke trials [30]. The TYR-TYRP1 transgene achieves adequate levels of expression and melanin synthesis to produce readily identifiable signal by in vivo MRI of stroked mice.

Nevertheless, this study has several shortcomings. The gene construct employed in this pilot study induced high, constitutive expression and was not designed for prolonged in vivo analysis. Previous studies have demonstrated that constitutive, high TYR-TRP expression reduces cell survival [17]. Further investigation is necessary to determine the appropriate level of transgene expression that produces measurable $\mathrm{T} 1$ shortening without altering transplant cell physiology. This may be accomplished by incorporating additional regulatory elements into the transgene construct, such as a Tet-On inducible promotor [31] or constitutively active promoters with lower protein expression such as ubiquitin and PGP [32]. Additionally, the results of a small sample size of five iPS NPC transplanted mice must be demonstrated in a larger cohort to show reproducibility.

Despite limitations, this study shows the feasibility of imaging clinically relevant stem cells in the brain utilizing an endogenous, positive contrast agent that is not prone to signal dilution with cell division. 
Additional research is warranted to investigate more nuanced approaches such as cell-type specific promoters for in vivo phenotyping of astrocyte (GFP A and Aldh1I [33, 34]) and neuronal (parvalbumin and CCK [35]) lineages. Further, inducible promoters [34,36] would allow control of a cell transplant biomarker at a specific time point, such as during an early phase of post-transplant stress or later period of functional integration.

\section{Conclusion}

MR imaging of clinically relevant cell lineage transplants through transgenic induction of melanogenesis is feasible. Further studies are needed to calibrate melanin synthesis to achieve non-toxic T1 shortening long term. These findings may prove useful to researchers investigating noninvasive treatment monitoring of cell based human therapies.

\section{Declarations}

\section{Author Contributions}

All authors contributed to the study conception and design. Material preparation, data collection and analysis were performed by ROC and JGJ. The first draft of the manuscript was written by ROC and JGJ, and all authors commented on previous versions of the manuscript. All authors read and approved the final manuscript.

\section{Additional Information}

The authors declare no competing interests.

\section{Data Availability}

The datasets generated during and/or analysed during the current study are available from the corresponding author on reasonable request.

\section{References}

1. Kalladka D, Sinden J, Pollock K, et al. Human neural stem cells in patients with chronic ischaemic stroke (PISCES): a phase 1, first-in-man study. Lancet. 388, 787-796. doi: 10.1016/S01406736(16)30513-X (2016).

2. Mack GS. ReNeuron and StemCells get green light for neural stem cell trials. Nat Biotechnol. 29, 9597 (2011).

3. Steinberg GK, Kondziolka D, Schwartz NE, et al. A novel phase 1/2A study of intraparenchymal transplantation of human modified bone marrow derived cells in patients with stable ischemic stroke. Stroke. 45, A149 (2014). 
4. Heslop JA, Hammond TG, Santeramo I, et al. Concise review: workshop review: understanding and assessing the risks of stem cell-based therapies. Stem Cells Transl Med. 4, 389-400 (2015).

5. Guenoun J, Ruggiero A, Doeswijk G, et al. In vivo quantitative assessment of cell viability of gadolinium or iron-labeled cells using MRI and bioluminescence imaging. Contrast Media Mol Imaging. 8, 165-174 (2013).

6. Xie J, Wang J, Niu G, et al. Human serum albumin coated iron oxide nanoparticles for efficient cell labeling. Chem. Commun. 46, 433-435 (2010).

7. Nguyen PK, Riegler J, Wu JC. Stem cell imaging: from bench to bedside. Cell Stem Cell. 14, 431-444 (2014).

8. Modo M, Mellodew K, Cash D, et al. Mapping transplanted stem cell migration after a stroke: a serial, in vivo magnetic resonance imaging study. Neuroimage. 21, 311-317 (2004).

9. Gambhir SS, Herschman HR, Cherry SR, et al. Imaging transgene expression with radionuclide imaging technologies. Neoplasia. 2, 118-138 (2000).

10. Zecca L, Bellei C, Costi P, et al. New melanic pigments in the human brain that accumulate in aging and block environmental toxic metals. Proc Natl Acad Sci U S A. 105, 17567-17572 (2008).

11. Castellanos G, Fernandez-Seara MA, Lorenzo-Betancor O, et al. Automated neuromelanin imaging as a diagnostic biomarker for Parkinson's disease. Mov Disord. 30, 945-952 (2015).

12. Sasaki M, Shibata E, Tohyama K, et al. Neuromelanin magnetic resonance imaging of locus ceruleus and substantia nigra in Parkinson's disease. Neuroreport. 17, 1215-1218 (2006).

13. Schwarz ST, Rittman T, Gontu V, Morgan PS, Bajaj N, Auer DP. T1-weighted MRI shows stagedependent substantia nigra signal loss in Parkinson's disease. Mov Disord. 26, 1633-1638 (2001).

14. Alfke H, Stoppler H, Nocken F, et al. In vitro MR imaging of regulated gene expression. Radiology. 228, 488-492 (2003).

15. Stritzker J, Kirscher L, Scadeng M, et al. Vaccinia virus-mediated melanin production allows MR and optoacoustic deep tissue imaging and laser-induced thermotherapy of cancer. Proc Natl Acad Sci US A. 110, 3316-3320 (2013).

16. Enochs WS, Petherick P, Bogdanova A, Mohr U, Weissleder R. Paramagnetic metal scavenging by melanin: MR imaging. Radiology. 204, 417-423 (1997).

17. Paproski RJ, Forbrich AE, Wachowicz K, Hitt MM, Zemp RJ. Tyrosinase as a dual reporter gene for both photoacoustic and magnetic resonance imaging. Biomed Opt Express. 2, 771-780 (2011).

18. Cockrell AS, Kafri T. Gene delivery by lentivirus vectors. Mol Biotechnol. 36, 184-204 (2007).

19. Sinn PL, Sauter SL, McCray PB, Jr. Gene therapy progress and prospects: development of improved lentiviral and retroviral vectors-design, biosafety, and production. Gene Ther. 12, 1089-1098 (2005).

20. Labat-gest V, Tomasi S. Photothrombotic ischemia: a minimally invasive and reproducible photochemical cortical lesion model for mouse stroke studies. J Vis Exp. 76, 50370 (2013).

21. IBM SPSS Statistics for Windows. Armonk, NY: IBM Corp. 2011; Version 20. 
22. Au P, Hursh DA, Lim A, et al. FDA oversight of cell therapy clinical trials. Sci Transl Med. 4, 149fs31 (2012).

23. Bhirde A, Xie J, Swierczewska M, Chen X. Nanoparticles for cell labeling. Nanoscale. 3, 142-153 (2011).

24. Shima T, Sarna T, Swartz HM, Stroppolo A, Gerbasi R, Zecca L. Binding of iron to neuromelanin of human substantia nigra and synthetic melanin: an electron paramagnetic resonance spectroscopy study. Free Radic Biol Med. 23, 110-119 (1997).

25. Zecca L, Pietra R, Goj C, Mecacci C, Radice D, Sabbioni E. Iron and other metals in neuromelanin, substantia nigra, and putamen of human brain. J Neurochem. 62, 1097-1101 (1994).

26. Prota G. Recent advances in the chemistry of melanogenesis in mammals. J Invest Dermatol. 75, 122-127 (1980).

27. Sulzer D, Bogulavsky J, Larsen KE, et al. Neuromelanin biosynthesis is driven by excess cytosolic catecholamines not accumulated by synaptic vesicles. Proc Natl Acad Sci U S A. 97, 11869-11874 (2000).

28. Jensen MB, Yan H, Krishnaney-Davison R, Al Sawaf A, Zhang SC. Survival and differentiation of transplanted neural stem cells derived from human induced pluripotent stem cells in a rat stroke model. J Stroke Cerebrovasc Dis. 22, 304-308 (2013).

29. Oki K, Tatarishvili J, Wood J, et al. Human-induced pluripotent stem cells form functional neurons and improve recovery after grafting in stroke-damaged brain. Stem Cells. 30, 1120-1133 (2012).

30. Azad TD, Veeravagu A, Steinberg GK. Neurorestoration after stroke. Neurosurgical Focus FOC. 40, E2 (2016).

31. Feng $H, X i a X, L i C$, et al. TYR as a multifunctional reporter gene regulated by the Tet-on system for multimodality imaging: an in vitro study. Sci Rep. 5, 15502 (2015).

32. Yu H, Fischer G, Jia G, Reiser J, Park F, Hogan QH. Lentiviral gene transfer into the dorsal root ganglion of adult rats. Mol Pain. 7, 63 (2011).

33. Kumar S, Zimmermann K, Hioki H, Pfeifer A, Baader SL. Efficient and graded gene expression in glia and neurons of primary cerebellar cultures transduced by lentiviral vectors. Histochem Cell Biol. 143, 109-121 (2015).

34. Liu B, Xu H, Paton JF, Kasparov S. Cell- and region-specific miR30-based gene knock-down with temporal control in the rat brain. BMC Mol Biol. 11, 93 (2010).

35. Mantoan Ritter L, Macdonald DC, Ritter G, et al. Lentiviral expression of GAD67 and CCK promoterdriven opsins to target interneurons in vitro and in vivo. J Gene Med. 18, 27-37 (2016).

36. Heinz N, Schambach A, Galla M, et al. Retroviral and transposon-based tet-regulated all-in-one vectors with reduced background expression and improved dynamic range. Hum Gene Ther. 22, 166176 (2011).

\section{Figures}




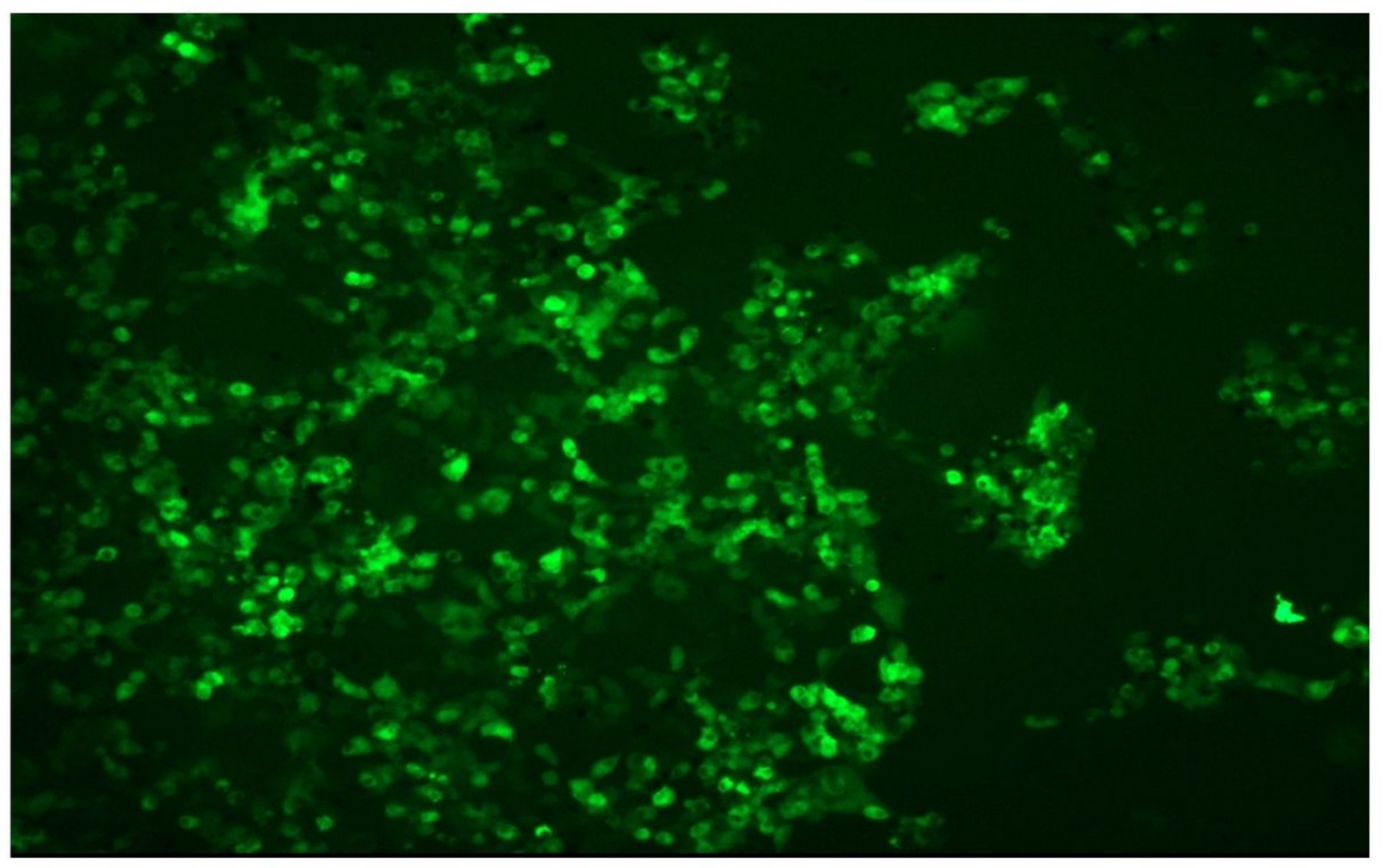

Figure 1

Fluorescent microscopy of transfected TYR TRY 293 HEK cells. The cells demonstrate in vitro GFP expression in excess of $50 \%$. 


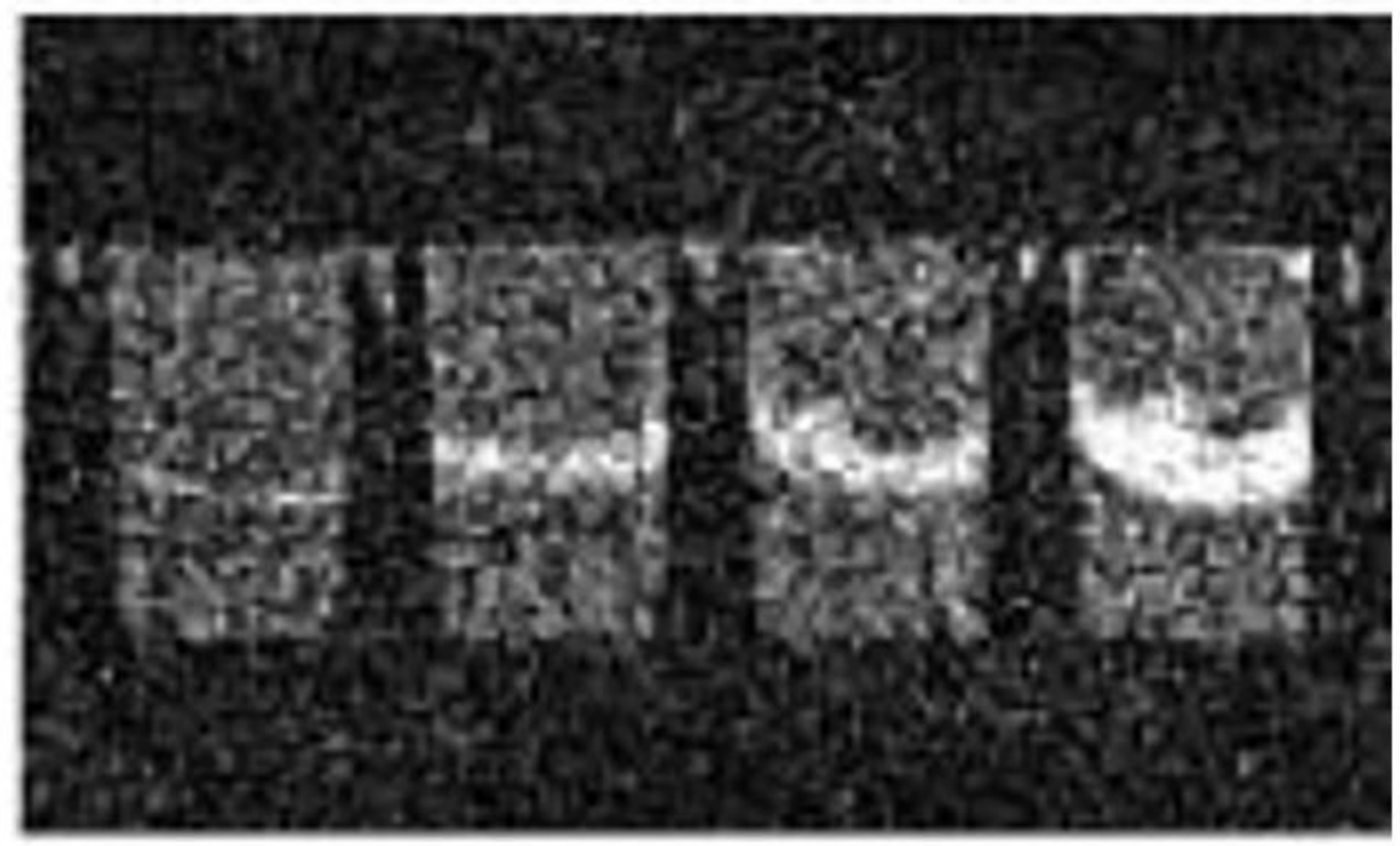

\section{Figure 2}

T1-weighted image of 24 well plate phantom containing transfected and nontransfected cells. The plate contains $5 \times 106$ transfected and nontransfected cells resuspended in agar at ratios of 0:1, 1:4, 1:1, and 1:0 (from left to right). 


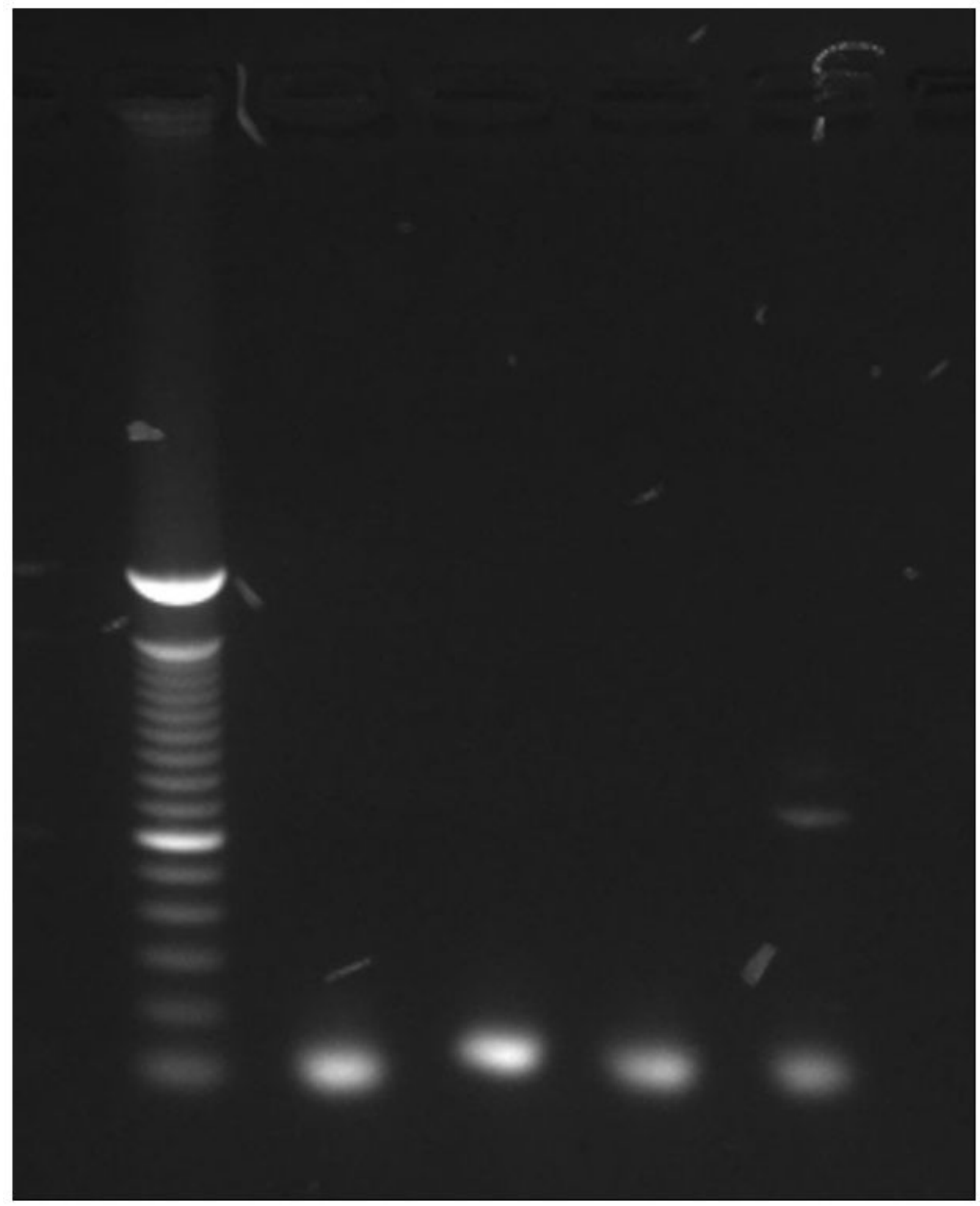

\section{Figure 3}

Agarose gel of control and transduced iPS NPC DNA following TYR TRP RT-PCR. In the leftmost column, a $600 \mathrm{bp}$ fragment corresponds to the tyrosinase mRNA transcript. 


\section{Figure 4}

In vitro fluorescent and light microscopy after staining. (a) In vitro fluorescent microscopy shows localization of mouse anti-human tyrosinase Ab (red) in transduced iPS NPCs cytosol. Cells were counterstained with DAPI (blue). (b) Light microscopy of the same cell population stained with FontanaMason for melanin (brown) and nuclear fast red for counterstain (pink). 


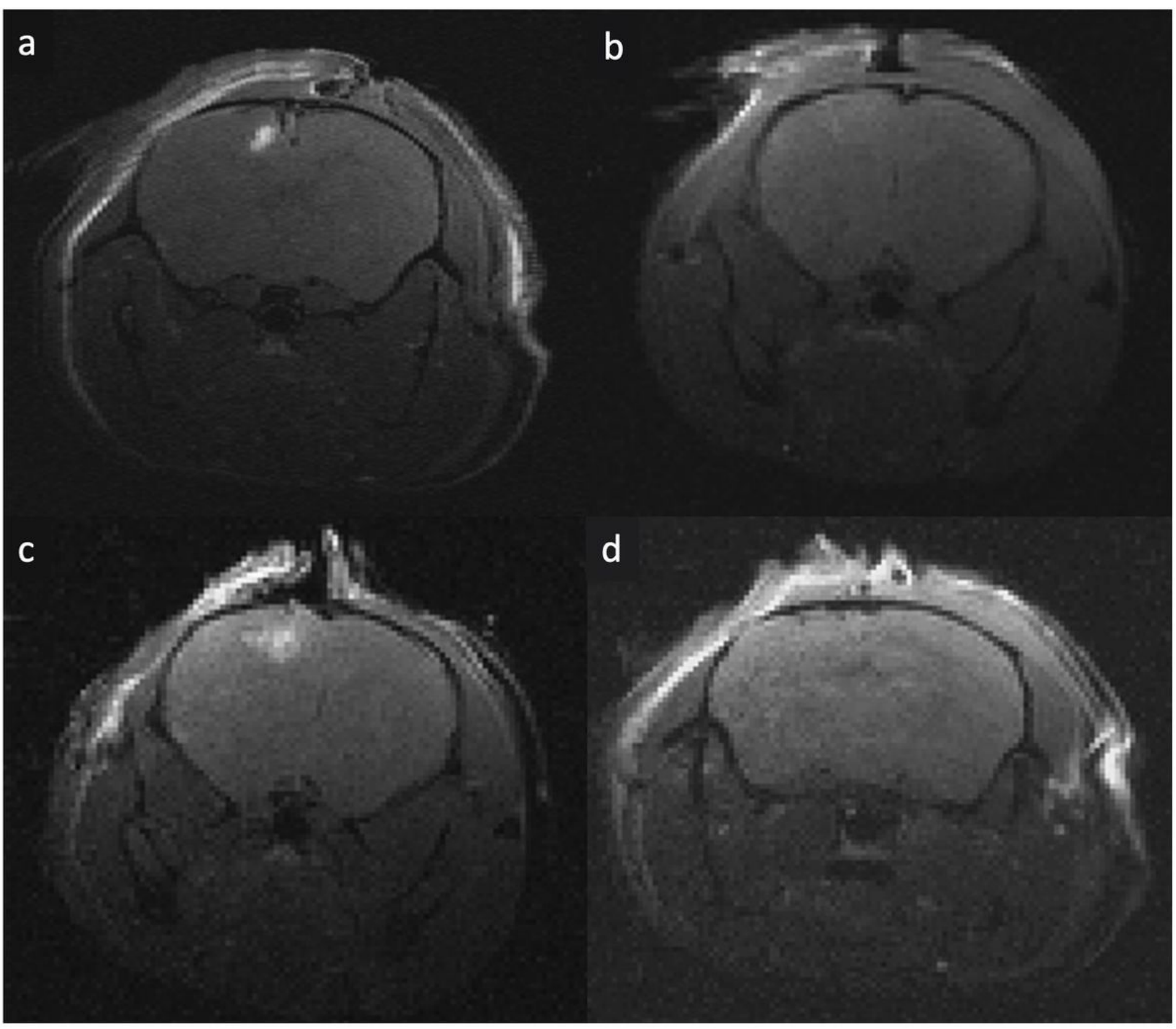

\section{Figure 5}

In vivo MRI of 293 HEK and iPS NPC cells. There is T1 shortening in TYR TRP (a) 293 HEK cells 1 day following IC injection into stroked mice (b) without corresponding signal in mice injected with nontransfected cells. (c) T1 shortening was similarly demonstrated in iPS NPC cells (d) without corresponding signal in mice injected with non-transduced cells. 


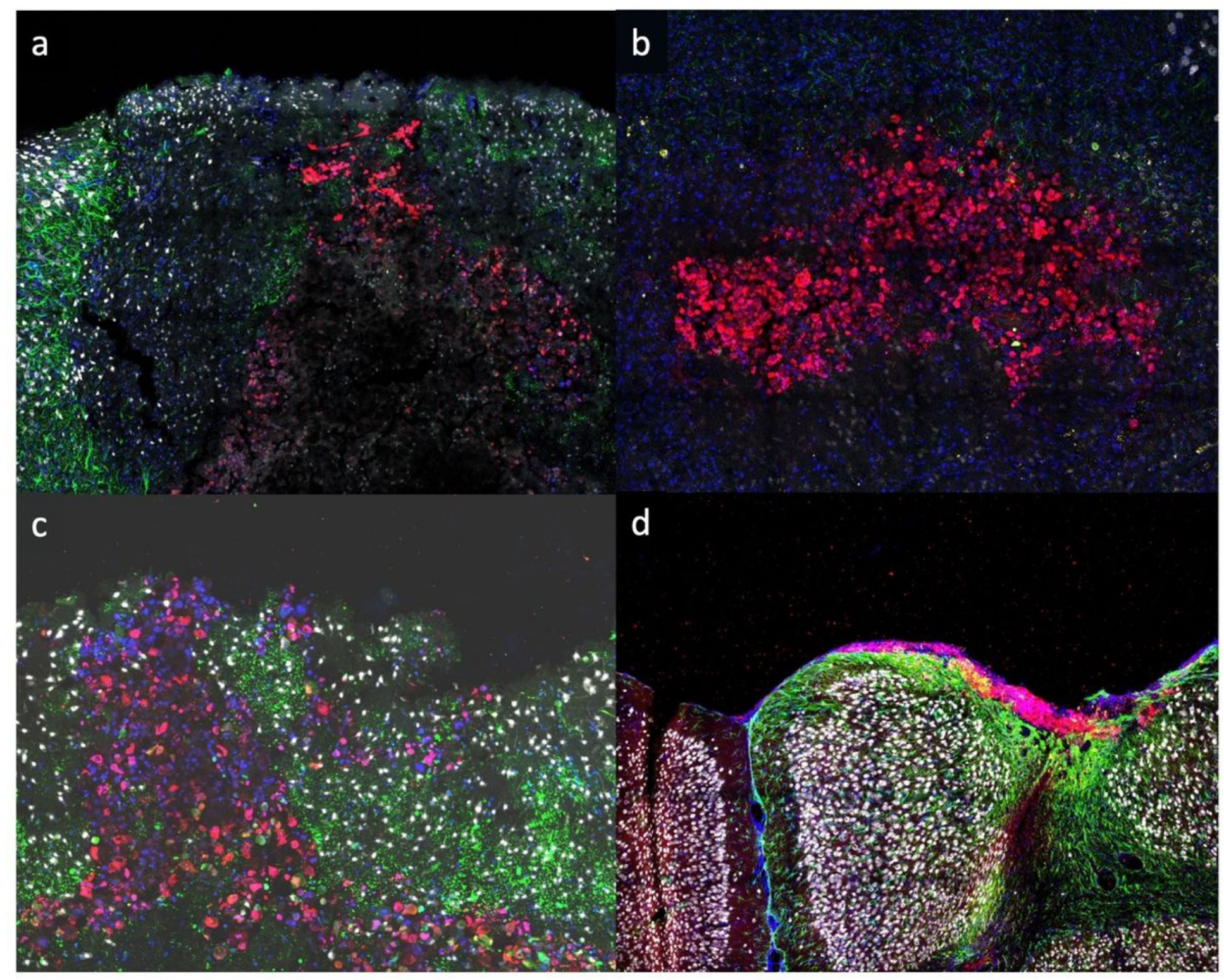

Figure 6

Histologic specimens through cell lines following IC injection sites 1 day after cell transplantation. (a) Melanic 293 HEK cells, (b) non-transfected native 293 HEK cells, (c) iPS NPC cells, and (d) nontransduced native iPS NPC cells are shown with corresponding antibody staining: green = GFAP; white $=$ NeuN; red = HuNu; and blue = DAPI . 